\title{
Perfil epidemiológico de mulheres atendidas na consulta do pré-natal de um hospital universitário
}

\author{
EPIDEMIOLOGICAL PROFILE OF CONSULTING WOMENAT THE PRENATAL \\ SERVICE OF A UNIVERSITY HOSPITAL
}

\author{
PERFIL EPIDEMIOLÓGICO DE MUJERES ATENDIDAS EN CONSULTA \\ PRENATAL DE UN HOSPITAL UNIVERSITARIO
}

Thelma Spindola ${ }^{1}$, Lúcia Helena Garcia Penna², Jane Márcia Progianti ${ }^{3}$

\section{RESUMO}

Trata-se de um estudo descritivo em abordagem quantitativa tendo como objeto as gestantes atendidas na consulta de pré-natal e o objetivo de identificar esta clientela traçando seu perfil epidemiológico. O Ambulatório de Obstetrícia de um Hospital público do município do Rio de Janeiro foi o cenário, onde foram investigadas 118 fichas de atendimento cadastradas de janeiro a junho de 2003. A análise dos resultados evidenciou que a maioria das mulheres estudadas concentra-se na faixa etária de 19 - 25 anos (39,8\%); casadas (38,9\%); com ensino fundamental $(29,6 \%)$; ocupação do lar $(33,8 \%)$; multíparas $(58,4 \%)$; iniciaram o prénatal com idade gestacional entre 14 e 17 semanas $(18,6 \%)$; tiveram anteriormente partos vaginais (79) e não referiram queixas na primeira consulta $(39 \%)$. Podemos concluir que o atendimento obstétrico contribui de maneira significativa para a redução da morbi-mortalidade das gestantes, possibilitando a orientação de intercorrências no ciclo grávidopuerperal e a prevenção de complicações.

\section{DESCRITORES}

Gravidez.

Saúde da mulher.

Enfermagem obstétrica.

\begin{abstract}
The characteristics of pregnant women in prenatal consultation are described in this article in a quantitative approach. The aim of this study is to identify this group by sketching its epidemiological profile. 118 attendance reports dated from January to June 2003 were investigated in the obstetrics service in a city of Rio de Janeiro public hospital. The analysis showed that the highest percentage is comprised of women in the age group of 19 25 years $(39,8 \%)$; that are married $(38,9 \%)$; have basic consultation between 14 and 17 weeks of pregnancy $(18,6 \%)$; had previous vaginal birth (79\%); have basic school education (29,6\%); are housewives $(33,8 \%)$; have more than one child $(58,4 \%)$; and did not mention complaints in the first consultation (39\%). It can be concluded that obstetric consultation can substantially help reduce morbi-mortality of pregnant women, since it makes possible the guiding of intercurrences and the prevention of troubles during and after the period of pregnancy.
\end{abstract}

\author{
KEY WORDS \\ Pregnancy. \\ Women's health. \\ Obstetrical nursing.
}

\section{RESUMEN}

Se trata de un estudio descriptivo con abordaje cuantitativo teniendo como objeto a gestantes atendidas en consulta prenatal y el objetivo de identificar a esta clientela trazando su perfil epidemiológico. El escenario fue el consultorio externo de obstetricia de un Hospital público del municipio de Rio de Janeiro, donde fueron investigadas 118 fichas de atención registradas de enero a junio del 2003. El análisis de los resultados evidenció que la mayoría de las mujeres estudiadas se concentra en el grupo etáreo de 19 25 años (39,8\%); casadas (38,9\%); con enseñaza fundamental $(29,6 \%)$; amas de casa (33,8\%); multíparas $(58,4 \%)$; iniciaron el prenatal con edad gestacional entre 14 y 17 semanas $(18,6 \%)$; tuvieron anteriormente partos vaginales (79\%) y no refirieron quejas en la primera consulta (39\%). Podemos concluir que la atención obstétrica contribuye de manera significativa en la reducción de la morbimortalidad de las gestantes, posibilitando la orientación de ocurrencias en el ciclo grávidopuerperal y la prevención de complicaciones.

\section{DESCRIPTORES}

Embarazo.

Salud de las mujeres.

Enfermería obstétrica.
1 Doutora em Enfermagem Professora Adjunta da Faculdade de Enfermagem da Universidade Estadual do Rio de Janeiro (UERJ).
Enfermeira Obsté- trica do HUGG da UNI-RIO. spindola@centroin. com.br
2 Doutora em Enfermagem. Professora Adjunta da Faculdade de Enfermagem da UERJ.
3 Doutora em Enfermagem. Professora Adjunta da Faculdade de Enfermagem da UERJ.




\section{INTRODUÇÃO}

O objeto deste estudo são as gestantes atendidas na consulta do pré-natal de um hospital universitário do município do Rio de Janeiro. O interesse pela temática emergiu da atividade assistencial de uma das pesquisadoras no Hospital Universitário Gaffrée e Guinle, da Universidade do Rio de Janeiro, e a vivência da implantação da consulta de enfermagem no prénatal. Nesta instituição, o acompanhamento das gestantes de baixo risco em conjunto com a equipe médica tornou-se efetivo a partir de 2004, com uma maior adesão das gestantes, embora tenha sido instituído em outubro de 2003. Desde 2000, a primeira consulta do pré-natal era realizada por enfermeiras que faziam a anamnese das gestantes e solicitavam exames de rotina, agendando as consultas subseqüentes com os médicos obstetras. Embora existisse o apoio das chefias (médica e enfermagem) havia muita resistência, por parte de alguns obstetras, para que as enfermeiras realizassem o acompanhamento no pré-natal de baixo risco, considerando que a instituição é referência para gestações de risco. Diante deste quadro, optou-se pela elaboração de uma pesquisa que permitisse identificar a clientela assistida delineando o seu perfil.

A gestação é classificada de baixo risco quando ela se constitui num fenômeno fisiológico normal que evolui, em 90\% dos casos, sem intercorrências; e de alto risco, quando já se inicia com problemas, ou estes surgem no decorrer do período com maior probabilidade de apresentarem uma evolução desfavorável, quer para o feto ou para a mãe ${ }^{(1)}$.

Chamamos a atenção que nestas clássicas denominações do modelo biomédico a gravidez é visualizada como um risco, mesmo quando é considerada normal e fisiológica pela sociedade medicalizada. Tal fato, em nosso entender, reflete uma concepção que submete este momento aos tratamentos intervencionistas fortalecendo a hegemonia médica no campo obstétrico e retira a autonomia do corpo feminino.

Um outro segmento da literatura específica reflete a experiência gestacional moderna, ampliando seu foco dos caracteres exclusivamente físicos para uma visão que inclui os aspectos psicossociais da gestação ${ }^{(2)}$. Neste sentido, os profissionais envolvidos na assistência obstétrica precisariam estar qualificados para reconhecer que as características sócio-econômicas e culturais das sociedades influenciam no desenvolvimento e nos cuidados do período gestacional, do parto e pós-parto.

Neste segmento mais ampliado está a enfermagem obstétrica, que considera a gestação uma experiência que envolve emoções, e tal como o parto, é um evento social que faz parte da sexualidade feminina e por isso mesmo, deve ser vivida com muito prazer. Por ser um momento de construção, algumas transformações poderão causar impactos de naturezas diversas, que podem variar dependendo da história de vida de cada mulher. Em nossa sociedade, as modificações desta construção são mais associadas aos aspectos negativos do que aos positivos.

Desta maneira, entendemos que as gestações fisiológicas, não possuem risco, portanto não deveriam ser classificadas de baixo risco, simplesmente deveríamos nos referir à elas como gestações fisiológicas. Quanto às gestações com problemas poderiam ser denominadas de gestações de risco.

O atendimento à mulher no ciclo grávido-puerperal é uma atividade prevista nas Ações Básicas da Assistência Integral à Saúde da Mulher preconizado pelo Ministério da Saú$\mathrm{de}^{(3)}$ e desenvolvida pelos profissionais de saúde nos hospitais e nos centros de saúde da rede básica.

Assim mesmo, cerca de 600 (seiscentas) mil mulheres morrem anualmente no mundo em decorrência de complicações da gravidez, parto e puerpério, sendo 99\% dessas mortes nos países subdesenvolvidos ou em desenvolvimento, entre os quais inclui-se o Brasil. A maioria dos óbitos relacionados diretamente com a função reprodutora "ocorrem devido a hipertensão na gravidez, à hemorragia, à infecção puerperal, a complicações no trabalho de parto e aborto"(4), situações evitáveis por meio de uma adequada assistência à mulher no ciclo gravídico-puerperal.

A assistência pré-natal tem como objetivo identificar adequada e precocemente quais as pacientes com mais chance de apresentar uma evolução desfavorável e acolher a mulher desde o início de sua gravidez ${ }^{(1)}$. O principal papel dos profissionais envolvidos neste atendimento é a escuta atentiva das clientes, transmitindo-lhes apoio e confiança necessários para que possam conduzir com autonomia suas gestações e partos. Neste sentido, é interessante que se efetuem trocas de experiências entre as mulheres e os profissionais de saúde - ações educativas - pois facilitam a compreensão do processo gestacional ${ }^{(3)}$. É através da educação que as mudanças sociais podem ocorrer, sendo cada encontro com o outro transformado num momento de troca, crescimento e resolução ${ }^{(5)}$.

A consulta de enfermagem obstétrica, nas gestações fisiológicas, tem sido realizada em muitas unidades básicas de saúde e hospitais municipais no Rio de Janeiro. De acordo com autores ${ }^{(6)}$ que estudam a temática, em meados dos anos 90, houve uma preocupação com a qualificação e ampliação do número de enfermeiras obstétricas da rede pública para atendimento do parto e pré-natal. O Ministério da Saúde, instituiu em 2000 o Programa de Humanização no Pré-natal e Nascimento no âmbito do SUS. A partir de 2001 desenvolve capacitações para Atenção Humanizada ao Parto/Nascimento destinada aos profissionais de saúde, criando um projeto de especialização de enfermeiras obstétricas $^{(7-9)}$. Todavia, em algumas instituições no município do Rio de Janeiro ainda existe muita resistência de médicos obstetras (especialmente nos hospitais universitários) para 
aceitar essa prática das enfermeiras, embora estas ações estejam amparadas pela lei do exercício profissional de enfermagem $^{(10)}$; Resolução COFEN no $271 / 2002^{(11)}$, no manual do pré-natal de baixo risco do Ministério da Saúde ${ }^{(3)}$ e Portarias 569 e 570 do Ministério da Saúde ${ }^{(7-8)}$.

Diante desta problemática, este trabalho tem como objetivos:

1- Identificar a clientela assistida na consulta de enfermagem do pré-natal.

2- Traçar o perfil epidemiológico das gestantes.

\section{METODOLOGIA}

Estudo do tipo exploratório descritivo em abordagem quantitativa, com emprego da técnica de observação indireta através da análise retrospectiva das fichas de atendimento do Serviço de Obstetrícia de uma instituição pública. Selecionou-se variáveis para compor o estudo do tipo: demográficas (faixa etária); sociais (escolaridade, estado civil, ocupação); biológicas e relacionadas com a história obstétrica (menarca, sexarca, métodos anticoncepcioanis, início pré-natal e outras).

O cenário do estudo foi o Hospital Universitário Gaffrée e Guinle (UNI-RIO) situado no município do Rio de Janeiro. Nele, a inclusão das gestantes no pré-natal é realizada pela enfermeira do Ambulatório de Obstetrícia, através de uma triagem onde são agendandas para a consulta de enfermagem as gestantes acima de 19 anos e que tenham idade gestacional até 20 semanas. Àquelas que não estão incluídas nos critérios de inclusão do serviço são encaminhadas para outras unidades de saúde, com exceção das adolescentes (atendidas em ambulatório próprio) e das cardiopatas, hipertensas ou com outras patologias associadas que são absorvidas, independente da idade gestacional, uma vez que a instituição é referência para o acompanhamento do alto risco obstétrico (nomenclatura convencional).

Nesta abordagem inicial são identificadas situações que necessitam de intervenção imediata como as perdas transvaginais, sangramentos, hipertensão e outras, procedendo-se o encaminhamento das gestantes para o prontoatendimento no Serviço de Obstetrícia, onde serão avaliadas pela equipe médica de plantão. A consulta de enfermagem obstétrica é realizada pela enfermeira do Ambulatório e por enfermeiras residentes, sendo atendidas de 10 a 12 gestantes semanalmente e agendadas com os obstetras para as consultas subseqüentes. Neste atendimento realiza-se a anamnese, obtendo informações acerca da história clínica e obstétrica da mulher, procede-se o exame físico e obstétrico, solicitam-se os exames laboratoriais de rotina no prénatal, sendo feitas as orientações de enfermagem pertinentes ao período gestacional e encaminhadas, se necessário, para demais especialidades como Dermatologia, Nutrição,
Psicologia e outros, ou para o pronto-atendimento na Obstetrícia.

A partir de outubro de 2003 teve início, para as gestantes fisiológicas (de baixo risco), a consulta obstétrica intercalada entre as equipes médica e de enfermagem. Nesta ocasião além dos procedimentos rotineiros no atendimento obstétrico (peso, aferição da pressão arterial, da altura uterina, ausculta do BCF, avaliação de exames e outros) são realizadas orientações pertinentes a cada período gestacional, bem como, àquelas relativas aos cuidados com o corpo, hábitos de vida, o parto e sinais de parto estimulando-se o diálogo e complementadas com a entrega de folderes explicativos.

Para início da coleta dos dados foi solicitada autorização à Comissão de Ética da Instituição de acordo com a Resolução 196 do Conselho Nacional de Saúde ${ }^{(12)}$. Foram analisadas 118 fichas de atendimento ambulatorial no período de janeiro a junho de 2003 com o auxílio de um roteiro de observação composto de 10 itens conforme as variáveis selecionadas. Os dados foram tabulados sendo aplicada a estatística descritiva, com emprego das freqüências absoluta e percentual, evidenciados em tabelas e discutidos a partir da Síntese de indicadores sociais ${ }^{(13-14)}$, de documentos da situação de saúde no Brasil ${ }^{(15)}$ e demais referências que analisam a temática.

\section{RESULTADOS E DISCUSSÃO}

A população estudada concentra-se na faixa etária de 19-25 anos (39,8\%) num total de 47 mulheres, seguidos de $38(32,2 \%)$ com idades de 26-30 anos(Tabela 1). Estes resultados estão em consonância com o Instituto Brasileiro de Geografia e Estatística ${ }^{(13)}$, determinando no último censo demográfico (2000) o total da população brasileira sendo 86.223.155 mulheres, sendo 46.881.521 com idade entre 15-49 anos, e destas 20.990 .967 concentram-se na região sudeste. Dos nascidos vivos em 2001, a idade das mães de maior prevalência é de 20-24 anos (771.480) e de 25-29 anos (585.181).

Tabela 1 - Distribuição das gestantes de acordo com a faixa etária - Rio de Janeiro - 2003

\begin{tabular}{lll}
\hline \multicolumn{1}{c}{ Faixa Etária } & Fi & F\% \\
\hline 19-25 anos & 47 & 39,8 \\
$26-30$ anos & 38 & 32,2 \\
$31-35$ anos & 20 & 16,9 \\
$36-40$ anos & 13 & 11,1 \\
\hline Total & $\mathbf{1 1 8}$ & 100 \\
\hline
\end{tabular}


De acordo com o Ministério da Saúde ${ }^{(15)}$ existe uma maior proporção de nascidos vivos no Brasil de mães cujas idades concentram-se entre os 15-34 anos. A região Sudeste apresenta uma proporção de 70,2\% de mães entre 20-34 anos, seguida da região Centro-Oeste $(68,6 \%)$ e da região Sul $(67,4 \%)$. Na faixa etária dos 15-19 anos a região Norte apresenta uma taxa de $28,9 \%$, a região Nordeste $24,9 \%$ e a Centro-Oeste $24,4 \%$.

Em relação ao estado civil, existe uma prevalência de casadas 46 (38,9\%) existindo, também, uma concentração significativa de mulheres que vivem em união estável $40(33,8 \%)$ ou solteiras 32 $(27,1 \%)$ (Tabela 2). Este resultado não confere com o censo demográfico de 2000, tendo evidenciado que os casamentos civis tiveram uma queda vertiginosa nas últimas décadas ${ }^{(13)}$. Em geral, as pessoas, pelos motivos mais variados, estão unindo-se consensualmente. Diante desta realidade o Código Civil Brasileiro, revisto e editado em janeiro de 2002, reconhece como entidade familiar a união estável entre homem e mulher configurada na convivência pública, contínua e duradoura e estabelecida com o objetivo de constituição de família ${ }^{(16)}$. Por outro lado, temos consciência que embora vivamos no século XXI, ainda existem muitas mulheres que omitem seu verdadeiro estado civil. No atendimento obstétrico onde ocorreu o estudo não é exigido nenhum documento, além do cartão de matrícula da instituição que não fornecia, até então, este tipo de informação.

Tabela 2 - Estado civil das gestantes atendidas no prénatal - Rio de Janeiro - 2003

\begin{tabular}{lcc}
\hline \multicolumn{1}{c}{ Estado Civil } & Fi & F\% \\
\hline Casada & 46 & 38,9 \\
União Consensual & 40 & 33,8 \\
Solteira & 32 & 27,1 \\
\hline Total & $\mathbf{1 1 8}$ & $\mathbf{1 0 0}$ \\
\hline
\end{tabular}

Quanto a escolaridade as gestantes têm, em sua maioria, o ensino fundamental 35 (29,6\%); $32(27,1 \%)$ mulheres têm o ensino fundamental incompleto e $32(27,1 \%)$ o ensino médio(Tabela 3). A baixa escolaridade do grupo pesquisado pode ser um agravante para a saúde das mulheres sendo considerado pelo Ministério da Saúde ${ }^{(3)}$ como um fator de risco obstétrico. Entretanto, dados do IBGE de 2003 apresentaram uma média superior de anos de estudo das mulheres em relação aos homens (7,0 contra 6,8). Refere que a população feminina busca uma maior escolaridade em função de transformações sócioeconômicas, havendo uma maior participação deste contingente populacional na renda das famílias ${ }^{(14)}$. Por outro lado, existe relação entre o grau de escolaridade da mãe e o número de consultas de pré-natal, conforme indicam dados do Ministério da Saúde ${ }^{(15)}$. Entre os nascidos vivos de mulheres que receberam sete ou mais consultas de pré-natal em 2001, o número de anos de estudo da mãe foi de quatro a sete anos $(34 \%)$ e oito a onze anos de instrução (33\%).
Tabela 3 - Escolaridade das gestantes atendidas no prénatal - Rio de Janeiro - 2003

\begin{tabular}{lrr}
\hline \multicolumn{1}{c}{ Escolaridade } & Fi & F\% \\
\hline Analfabeta & 1 & 0,8 \\
Ensino Fundamental & 35 & 29,6 \\
Fundamental Incompleto & 32 & 27,1 \\
Ensino Médio & 32 & 27,1 \\
Ens. Médio Incompleto & 12 & 10,1 \\
Superior & 4 & 3,3 \\
Superior Incompleto & 2 & 1,6 \\
\hline Total & $\mathbf{1 1 8}$ & $\mathbf{1 0 0}$ \\
\hline
\end{tabular}

Quanto a profissão/ocupação das gestantes $40(33,8 \%)$ não exercem atividade remunerada, $19(16,1 \%)$ são empregadas doméstica e $10(8,4 \%)$ são vendedoras(Tabela 4). Esses resultados tornam evidentes que em função da baixa escolaridade as gestantes ou não exercem atividade remunerada ou trabalham em atividades do setor terciário da economia, no ramo do trabalho doméstico. Esses dados estão em consonância com estudiosos da inserção da mulher no mercado de trabalho ${ }^{(17-18)}$. A autora $^{(18)}$ acrescenta que as mulheres trabalhadoras concentram-se em verdadeiros guetos ocupa-cionais em atividades com pouco ou nenhum prestígio social, como, por exemplo, a atividade doméstica. $\mathrm{O}$ que interfere na participação das mulheres no mercado de trabalho são a responsabilidade com a guarda, cuidado e educação dos filhos na família, além da insuficiência de creches e dos rendimentos obtidos serem insuficientes para cobrir os gastos de sua saída de casa ${ }^{(19)}$. Dados do $\mathrm{IBGE}^{(14)}$ confirmam a desigualdade de rendimentos entre homens e mulheres, sendo maior nos níveis de escolaridade de até três anos de grau de instrução e de 8 a 10 anos de estudo.

Tabela 4 - Distribuição das gestantes conforme a profissão/ocupação - Rio de Janeiro - 2003

\begin{tabular}{lcc}
\hline \multicolumn{1}{c}{ Profissão/ocupação } & Fi & F\% \\
\hline Do lar & 40 & 33,8 \\
Empregada Doméstica & 19 & 16,1 \\
Vendedora & 10 & 8,4 \\
Aux. Administrativo & 8 & 6,7 \\
Aux. Serviços Gerais & 6 & 5,0 \\
Professora & 5 & 4,2 \\
Balconista & 5 & 4,2 \\
Operadora de Caixa & 5 & 4,2 \\
Estudante & 4 & 3,3 \\
Téc. Enfermagem & 3 & 2,5 \\
Comerciante & 2 & 1,6 \\
Costureira & 2 & 1,6 \\
Manicure & 2 & 1,6 \\
Cozinheira & 2 & 1,6 \\
Esteticista & 2 & 1,6 \\
Biológa & 1 & 0,8 \\
Recepcionista & 1 & 0,8 \\
Industriaria & 1 & 0,8 \\
\hline Total & $\mathbf{1 1 8}$ & $\mathbf{1 0 0}$ \\
\hline
\end{tabular}


A distribuição das mulheres em relação a menarca evidenciou que a maioria $73(61,8 \%)$ teve o início do ciclo menstrual entre 12-14 anos; 21 (17,7\%) entre 9-11 anos e 15-18 anos respectivamente (Tabela 5).

Tabela 5 - Distribuição das gestantes conforme a menarca Rio de Janeiro - 2003

\begin{tabular}{lcc}
\multicolumn{1}{c}{ Menarca } & Fi & F\% \\
\hline 09-11 anos & 21 & 17,7 \\
12-14 anos & 73 & 61,8 \\
15-18 anos & 21 & 17,7 \\
Não sabia informar & 3 & 2,5 \\
\hline Total & 118 & 100 \\
\hline
\end{tabular}

A sexarca ocorreu entre 16-20 anos em 64 (54,2\%) gestantes e 12-15 anos em 22 (18,6\%) (Tabela 6).

Tabela 6 - Distribuição das gestantes conforme a sexarca Rio de Janeiro - 2003

\begin{tabular}{lrr}
\multicolumn{1}{c}{ Sexarca } & Fi & F\% \\
\hline 12-15 anos & 22 & 18,6 \\
16-20 anos & 64 & 54,2 \\
$21-25$ anos & 21 & 17,7 \\
26-30 anos & 5 & 4,2 \\
Acima de 30 anos & 1 & 0,8 \\
Não sabia informar & 3 & 2,5 \\
\hline Total & 118 & 100 \\
\hline
\end{tabular}

Este dado é relevante quando analisarmos a relação entre menarca e sexarca destas mulheres observando que existe uma correlação entre esses dados. Atualmente, as jovens têm iniciado precocemente a vida sexualmente ativa, o que tem aumentado, de maneira significativa, os riscos de contraírem uma doença sexualmente transmissível (DST) e/ou uma gravidez não planejada ${ }^{(20)}$. Este aspecto deve ser trabalhado com as gestantes sendo orientadas quanto a prevenção das DST e o planejamento das gestações futuras. Segundo o Ministério da Saúde ${ }^{(15)}$, em 2001 o Sisnac registrou um total de 3.106.525 nascidos vivos, sendo $723.070(23,3 \%)$ filhos de mães adolescentes (10-19 anos). A região Norte apresentou a maior proporção de nascidos vivos de mães com idade entre $10-14$ anos $(1,4 \%)$, a região Sudeste foi a que apresentou a menor proporção neste grupo de idade $(0,6 \%)$. O estado do Maranhão foi o que apresentou a maior proporção $(31,9 \%)$ de nascidos vivos de mães adolescentes (10-19 anos), seguido por Tocantins $(31,6 \%)$. As menores proporções nesta faixa etária foram encontradas no Distrito Federal $(18,7 \%)$, São Paulo $(19,1 \%)$ e Rio Grande do Sul $(20,2 \%)$.

Em relação aos métodos contraceptivos utilizados pelas mulheres, a maioria $54(45,7 \%)$ faz uso de anticoncepcionais hormonais orais e $47(39,8 \%)$ não utilizam nenhum método(Tabela 7).

Tabela 7 - Método contraceptivo utilizado pelas mulheres Rio de Janeiro - 2003

\begin{tabular}{lrr}
\hline \multicolumn{1}{c}{ Método } & Fi & F\% \\
\hline Hormônio oral & 54 & 45,7 \\
Nenhum & 47 & 39,8 \\
Preservativo Masc. & 10 & 8,4 \\
Hormônio Injetável & 3 & 2,5 \\
Tabela & 2 & 1,6 \\
Coito interrompido & 2 & 1,6 \\
\hline Total & $\mathbf{1 1 8}$ & $\mathbf{1 0 0}$
\end{tabular}

Este aspecto é relevante e deve ser valorizado pelos profissionais de saúde no acompanhamento destas mulheres, uma vez que a falta de orientação em relação a contracepção é um dos fatores responsável pelas gravidezes indesejadas. Durante a realização do pré-natal é importante que sejam estimuladas a comparecerem para a revisão pós-parto e serem orientadas em relação a contracepção. Vale salientar, todavia, que existe nas Unidades Básicas de Saúde do Município do Rio de Janeiro o Serviço de Planejamento Familiar onde são avaliadas por um ginecologista, fazem exame colpocitológico e recebem orientações em grupo relativas aos diversos métodos contraceptivos (efeitos no organismo, vantagens e desvantagens), possibilitando que escolham o método e possam recebê-lo gratuitamente (anticoncepcional hormonal oral, preservativo, diafragma, DIU e espermicida $^{(21)}$.

As gestantes são multíparas $69(58,4 \%)$ e nulíparas $49(41,5 \%)$ sendo, na maioria dos casos, a segunda ou terceira gestação que estava evoluindo. Estes dados são corroborados pelo $\mathrm{IBGE}^{(13)}$ evidenciando em 2001 a existência no Brasil de 46.881.521 mulheres com idade entre 15-49 anos. A região sudeste concentra 20.990 .967 com 2.108 .168 nascidos vivos, sendo $4.6 \%$ filho, $0.6 \% 2$ filhos e $0.1 \% 3$ filhos. A taxa de fecundidade total é de $2,18 \%$ e de natalidade $19,89 \%{ }^{(13)}$. De acordo com o Ministério da Saúde ${ }^{(15)}$ houve uma redução em $50 \%$ na taxa de fecundidade total em todas as regiões brasileiras no período de 1970 a 2000 . A maior redução foi na região Norte passando de 8,2 filhos por mulher em 1970 para 3,2 em 2000. Considerando as taxas específicas de fecundidade por grupos específicos de idade das mulheres no período reprodutivo, o único grupo que teve aumento nesse período foi o de 15 a 19 anos de idade ${ }^{(15)}$.

A distribuição das mulheres pela idade gestacional em que iniciaram o pré-natal revelou que a maioria $22(18,6 \%)$ teve sua primeira consulta entre 14 e 17 semanas, seguidas de $19(16,1 \%)$ que tiveram entre 17 semanas e 01 dia e 20 semanas e 11 semanas e 01 dia e 14 semanas, respectivamente (Tabela 8). 
Tabela 8 - Idade gestacional em que as mulheres iniciaram o pré-natal - Rio de Janeiro - 2003

\begin{tabular}{lrr}
\hline \multicolumn{1}{c}{ Idade gestacional } & Fi & F\% \\
\hline 07-11 semanas & 9 & 7,6 \\
$11 \mathrm{~s}+01 \mathrm{~d}-14 \mathrm{~s}$ & 19 & 16,1 \\
$14 \mathrm{~s}+01 \mathrm{~d}-17 \mathrm{~s}$ & 22 & 18,6 \\
$17 \mathrm{~s}+01 \mathrm{~d}-20 \mathrm{~s}$ & 19 & 16,1 \\
$20 \mathrm{~s}+01 \mathrm{~d}-23 \mathrm{~s}$ & 14 & 11,8 \\
$23 \mathrm{~s}+01 \mathrm{~d}-26 \mathrm{~s}$ & 11 & 9,3 \\
Acima de 26 semanas & 10 & 8,4 \\
Não há registro & 14 & 11,8 \\
\hline Total & $\mathbf{1 1 8}$ & $\mathbf{1 0 0}$
\end{tabular}

Este dado é relevante para a assistência obstétrica considerando que o manual do Ministério da Saúde ${ }^{(3)}$ e as Portarias 569 e $570^{(7-8)}$ recomendam que toda gestante tenha pelo menos 6 (seis) consultas no período gestacional. Um dos objetivos do acompanhamento no pré-natal é o acolhimento desta mulher desde o início de sua gravidez. Este atendimento deve ocorrer de preferência no primeiro trimestre, com o propósito de que se realizem intervenções oportunas durante o período gestacional, sejam elas preventivas ou terapêuticas ${ }^{(3,7-8)}$. Todavia, devemos reconhecer possíveis falhas no sistema de captação destas mulheres para o atendimento obstétrico. Na instituição em que o estudo ocorreu as gestantes, em muitas situações, referem tentativas frustradas de atendimento pré-natal em outras instituições de saúde do município. Em geral, as unidades procuram organizar a captação de mulheres priorizando o atendimento para as gestações de risco e adolescentes, o que acaba dificultando o acesso ao programa para àquelas que não preenchem estes requisitos. Por outro lado, para que se estabeleça um elo de ligação entre os profissionais de saúde e as gestantes é importante que se inicie precocemente o acompanhamento no pré-natal, quando cria-se possibilidades para que se estabeleça uma relação de confiança e uma participação efetiva a cada momento vivenciado no período gestacional.

Outro dado relevante nesta tabela é a falta de registro da idade gestacional com $14(11,8 \%)$ ocorrências. Neste sentido, vale acrescentar que, em algumas situações, as mulheres não sabem informar a data da última menstruação (DUM) - por lapso de memória ou ausência de amenorréia - dificultando e/ou impedindo a estimativa da idade gestacional, sendo necessário utilizar outros recursos para sua determinação.

A história obstétrica das gestantes revelou que entre as multíparas (69) ocorreram registros de 79 partos vaginais e 39 partos cesáreos, considerando todas as gravidezes. Entre as patologias associadas 12 apresentaram Doença Hipertensiva Específica da Gestação (DHEG); 05 préeclâmpsia; 01 eclâmpsia e 01 Diabetes Gestacional. De acor- do com o Ministério da Saúde ${ }^{(22)}$, as taxas de cesariana apresentadas ao Sistema Único de Saúde tiveram um declínio nos últimos anos, tendo atingido 24\% no ano de 2000. O acompanhamento da mulher no pré-natal é preponderante para a detecção e prevenção de complicações obstétricas através de uma intervenção oportuna. Conhecer a história da gestante e suas experiências anteriores permite ao profissional de saúde perceber a mulher de maneira única, com suas peculiaridades, receios e dúvidas, assumindo importância neste contexto as orientações obstétricas e o envolvimento que contribuem para aumentar a auto-estima da mulher e o controle do processo de gestar ${ }^{(22)}$. Considerando que o estudo ocorreu numa instituição especializada no atendimento de gestações de risco, do conjunto amostral apenas $16 \%$ tiveram manifestações que configuravam situações de risco obstétrico.

Em relação às queixas registradas no primeiro atendimento obstétrico a maioria (39) não referiu queixas; 27 relataram dor em baixo ventre sem perdas transvaginais; 23 náuseas e 15 cefaléia não associada a Hipertensão Arterial (Quadro 1). Estes dados evidenciam o perfil da clientela - gestantes fisiológicas- e corroboram a importância da assistência pré-natal que compreende todas as medidas introduzidas e/ou recomendadas (diagnósticas, preventivas e curativas) durante a gestação com objetivo de preservar a saúde materno-fetal e reduzir a morbi-mortalidade do binômio mãe-filho ${ }^{(23)}$.

Quadro 1 - Principais queixas referidas pelas gestantes - Rio de Janeiro - 2003

\begin{tabular}{|lc|}
\hline \multicolumn{1}{|c|}{ Queixas } & Fi \\
\hline Não referiu queixas & 39 \\
Dor baixo ventre s/perdas & 27 \\
Náuseas & 23 \\
Cefaléia & 15 \\
Emese & 9 \\
Prurido Vaginal & 9 \\
Perdas transvaginais & 8 \\
Constipação & 6 \\
Fadiga & 3 \\
Disúria & 2 \\
\hline
\end{tabular}

\section{CONCLUSÕES}

Este estudo em abordagem quantitativa foi resultado da participação de enfermeiras no atendimento às gestantes, através da implementação da consulta de enfermagem obstétrica, no Hospital Universitário Gafrée e Guinle (HUGG) no Rio de Janeiro. Considerando tratar-se de uma instituição referência para o acompanhamento de gestações de risco, houve o interesse em traçar o perfil da clientela assistida, acreditando-se que poderíamos, então, reconhecer suas características e identificar fatores de risco no processo de gestar e intercorrências que poderiam ser evitadas ou tratadas. 
O perfil das gestantes atendidas é de mulheres jovens, com baixa escolaridade, casadas, que não exercem atividade remunerada, multíparas, que iniciaram o pré-natal no segundo trimestre por não conseguirem vaga para esta modalidade de atendimento em outras instituições públicas, ou seja dependentes do Sistema de Saúde vigente no país e com gravidez fisiológica (de baixo risco). A maioria não referiu queixas na primeira consulta e não apresentava patologias associadas ao processo gestacional. Do conjunto amostral (118 mulheres), apenas $16 \%$ das multíparas tiveram manifestações em gestações anteriores que configuravam risco obstétrico.

A prevenção de complicações obstétricas e as ações educativas desenvolvidas no pré-natal são essenciais para o acompanhamento e orientação da mulher no ciclo grávidopuerperal, ressaltando-se o papel preponderante da enfermagem obstétrica neste processo. É interessante salientar que, em muitas unidades básicas de atenção à saúde no Rio de Janeiro, as enfermeiras obstétricas, têm participado no

\section{REFERÊNCIAS}

(1) Buchabqui JA, Abeche AM, Brietzke E. Assistência pré-natal. In: Rotinas em obstetrícia. $4^{\mathrm{a}}$ ed. Porto Alegre: Artmed; 2001 p. 23-37.

(2) Burroughs A. Uma introdução à enfermagem materna. Porto Alegre: Artes Médicas; 1995.

(3) Brasil. Ministério da Saúde. Assistência pré-matal: manual técnico. Brasília: Secretaria de Políticas de Saúde; 2000.

(4) Lacava RMVB, Barros SMO. Prática de enfermagem durante a gravidez. In: Barros SMO, Marin HF, Abrão ACFV. Enfermagem obstétrica e ginecológica: guia para a prática assistencial. São Paulo: Roca; 2002. p. 116-41.

(5) Santi MC. Dimensão educadora na assistência de enfermagem obstétrica e ginecológica. In: Barros SMO, Marin HF, Abrão ACFV. Enfermagem obstétrica e ginecológica: guia para a prática assistencial. São Paulo: Roca; 2002. p. 502-11.

(6) Vargens, OMC, Progianti, JM. O processo de desmedicalização da assistência à mulher no ensino da enfermagem. Rev Esc Enferm USP. 2004;38(1):46-50.

(7) Brasil. Ministério da Saúde. Portaria n. 569, de 01 de junho de 2000. Institui o Programa de Humanização no Pré-natal e Nascimento no âmbito do SUS. [online]. Disponível em: http:// dtr 2001.saude.gov.br/sas/PORTARIAS/PORT 2000/GM/GM569 rep.htm [Acesso em 24 jul. 2005].

(8) Brasil. Ministério da Saúde. Portaria n. 570, de 01 de junho de 2000. Institui o Componente I do Programa de Humanização no Pré-natal e Nascimento: incentivo à assistência pré-natal no âmbito do Sistema Único de Saúde. [online]. Disponível em: http:// dtr 2001.saude.gov.br/sas/PORTARIAS/PORT 2000/ GM/GM-569 rep.htm [Acesso em 24 jul. 2005]. acompanhamento das gestações fisiológicas conforme as recomendações do manual do Ministério da Saúde ${ }^{(3,7-8)}$.

No HUGG, a partir de 2004, intensificou-se a participação das enfermeiras no acompanhamento das gestantes fisiológicas (baixo risco) numa atuação em conjunto com a equipe médica (são atendidas pelas duas equipes). Das 653 gestantes atendidas no pré-natal, em 2004, houve participação das enfermeiras em 364 atendimentos de primeira vez e 289 atendimentos subseqüentes. Sabe-se que, embora as enfermeiras obstétricas tenham amparo legal ${ }^{(3,7-}$ 8,10-11) para o exercício de suas atividades, a resistência de alguns obstetras no município do Rio de Janeiro ainda se faz presente. Entretanto, na rede municipal tem tido um terreno fértil para sua atuação. Neste hospital achamos oportuno, em princípio, caracterizar a população e a partir dos resultados gradati-vamente as enfermeiras, foram ampliando suas ações e vencendo resistências pela realização de um trabalho consciente, criterioso, considerando tratar-se de um hospital especializado em gestações de risco.

(9) Brasil. Ministério da Saúde. Taxa de mortalidade materna cai 28\%. 19/12/2002. [online]. Disponível em: http:// portalweb02. saude.gov.br/portal/aplicaçoes/busca/buscar.cfm? [Acesso em 24 jul. 2005].

(10) Brasil. Decreto-lei n. 94.406/87, de 08 de junho de 1987. Regulamenta a Lei 7498/86 que dispõe sobre o Exercício da Enfermagem e dá outras providências. Diário Oficial da República Federativa do Brasil, Brasília, 09 jun. 1987. Seção I, p. 8853-5.

(11) Conselho Federal de Enfermagem. Resolução COFEN n. 271/ 2002. Regulamenta ações do enfermeiro na consulta, prescrição de medicamentos e requisição de exames. [online]. Disponível em: http://www.portalcofen.com.br [Acesso em 18 jul. 2005].

(12) Brasil. Ministério da Saúde. Fundação Oswaldo Cruz. Resolução 196/96 do Conselho Nacional de Saúde. Diretrizes e normas regulamentadoras de pesquisas envolvendo seres humanos. Brasília; 1996.

(13) Fundação Instituto Brasileiro de Geografia e Estatística (IBGE). Censo demográfico 2000. [online] Disponível em: http:// www.ibge.gov.br/indicadores_sociais/sintese_de_indicadores_ sociais_2002. [Acesso em 12 nov. 2003].

(14) Fundação Instituto Brasileiro de Geografia e Estatística (IBGE). Departamento de População e Indicadores Sociais. Síntese de indicadores sociais. Rio de Janeiro: IBGE; 2004.

(15) Brasil. Ministério da Saúde. Secretaria de Vigilância em Saúde. Departamento de Análise de Situação de Saúde. Saúde Brasil 2004: uma análise da situação de saúde. Brasília; 2004. 
(16) Brasil. Lei n. 10.406, de 10 de janeiro de 2002. Institui o Código Civil. [online]. Disponível em: http:// www.terra.com.br/notícias/codigocivil_integra.zip. [Acesso em 12 nov. 2003].

(17) Rosemberg F. A educação de mulheres jovens e adultas no Brasil. In: Saffioti HIB, Vargas M, organizadoras. Mulher brasileira é assim. Rio de Janeiro: Rosa dos Tempos; 1994. p. 27-62.

(18) Fonseca RMGS. Atenção: mulheres trabalhando! (na vida, na saúde, na enfermagem). In: Fonseca RMGS, organizadora. Mulher e cidadania na nova ordem social. São Paulo: NEMGE/ USP; 1996. p. 97-110.

(19) Bruschini C. O trabalho da mulher no Brasil: tendências recentes. In: Saffioti HIB, Vargas M. organizadoras. Mulher brasileira é assim. Rio de Janeiro: Rosa dos Tempos; 1994. p. 63-93.
(20) Takiuti AD. A saúde da mulher adolescente - 1993. In: Madeira FR. Quem mandou nascer mulher? Estudos sobre crianças e adolescentes pobres no Brasil. Rio de Janeiro: Record/Rosa dos Tempos; 1997. p. 213-90.

(21) Valladares DP. Ações de contracepção e assistência ao parto: a experiência do Rio de Janeiro. In: Giffin K, Costa SH, organizadoras. Questões da saúde reprodutiva. Rio de Janeiro: Fiocruz; 1999. p. 357-94.

(22) Brasil. Ministério da Saúde. Secretaria de Políticas de Saúde. Área Técnica de Saúde da Mulher. Parto, aborto e puerpério: assistência humanizada à mulher. Brasília; 2001.

(23) Neme B, Maretti M. Assistência pré-natal. In: Neme B. Obstetrícia básica. $2^{\mathrm{a}}$ ed. São Paulo: Sarvier; 2000. p. 117-33. 